\title{
Mesenchymal Stem Cell Secretome for Dermatology Application: A Review
}

\author{
Restu Harisma Damayanti iD \\ Taofik Rusdiana iD \\ Nasrul Wathoni iD \\ Department of Pharmaceutics and \\ Pharmaceutical Technology, Faculty of \\ Pharmacy, Universitas Padjadjaran, \\ Sumedang, 45353, Indonesia
}

\begin{abstract}
Secretome, also known as conditioned medium, is a secreted molecule from mesenchymal stem cells (MSCs) that has a variety of biological activities that can be used in various therapies, especially on the skin applications. A lack of conventional therapies makes secretome as a promising alternative therapy. The presence of growth factors, cytokines, and extracellular vesicles including microvesicles and exosomes in secretome has been widely reported, which serves in improving the proliferation and migration of cells to help in skin regeneration. Therefore, we were able to optimize the use of this secretome in a well-needed special review related to its work in addressing various skin problems. So, in this article, we discussed the benefits and biological activity of secretome on the skin application. This review was compiled based on the approval of several sites, such as Scopus, PubMed, Science Direct, and Google Scholar with the terms "MSC secretome for skin," "secretome for skin," "secretome dermatology," "secretome conditioned medium for skin," "secretome conditioned medium for skin wound," "secretome conditioned medium for aging," "secretome conditioned medium for hair growth," and "secretome conditioned medium for psoriasis." A total of 215 articles were collected for selection, of which 90 articles were used. Based on the results, it was concluded that secretome has a variety of useful activities to regenerate and repair tissue damage that have not been used on the skin, such as for wound healing, photoprotection, promotion of hair growth, psoriasis treatment, and other application as antimicrobial.
\end{abstract}

Keywords: MSC secretome, conditioned medium, biological activity, skin application

\section{Introduction}

The skin is the largest organ of the body and serves as the first defense against various disorders and external stimuli causing susceptibility to infection and inflammation. ${ }^{1}$ Various skin problems are often found, ranging from acute to chronic skin disorders that can impair the structure and function of the skin to triggering various reactions on the skin. In chronic skin conditions, such as infection, inflammation, ultraviolet (UV) exposure and others, general therapy is not able to restore the function and structure of injured skin tissue under normal circumstances. Thus, triggering the development of more effective therapies. ${ }^{2}$

The development of stem cell-based therapies continues to today, especially in skin disease therapy. Stem cells, in particular mesenchymal stem cells (MSCs), can secrete trophic factors responsible for regenerating tissue and repairing tissue damage. $^{3-5}$ However, this therapy using MSCs shows low cell survival and engraftment rates after the transplant process, cell retention, and, in case of mutations from stem cells, can lead to cancer. ${ }^{1,2,6,7}$ The main mechanism of action of MSCs is
Correspondence: Nasrul Wathoni Department of Pharmaceutics and Pharmaceutical Technology, Faculty of Pharmacy, Universitas Padjadjaran, Sumedang, 45353, Indonesia

Tel +6222842888888

Email nasrul@unpad.ac.id 


\section{Graphical Abstract}

\section{EFFECTS FOR SKIN}

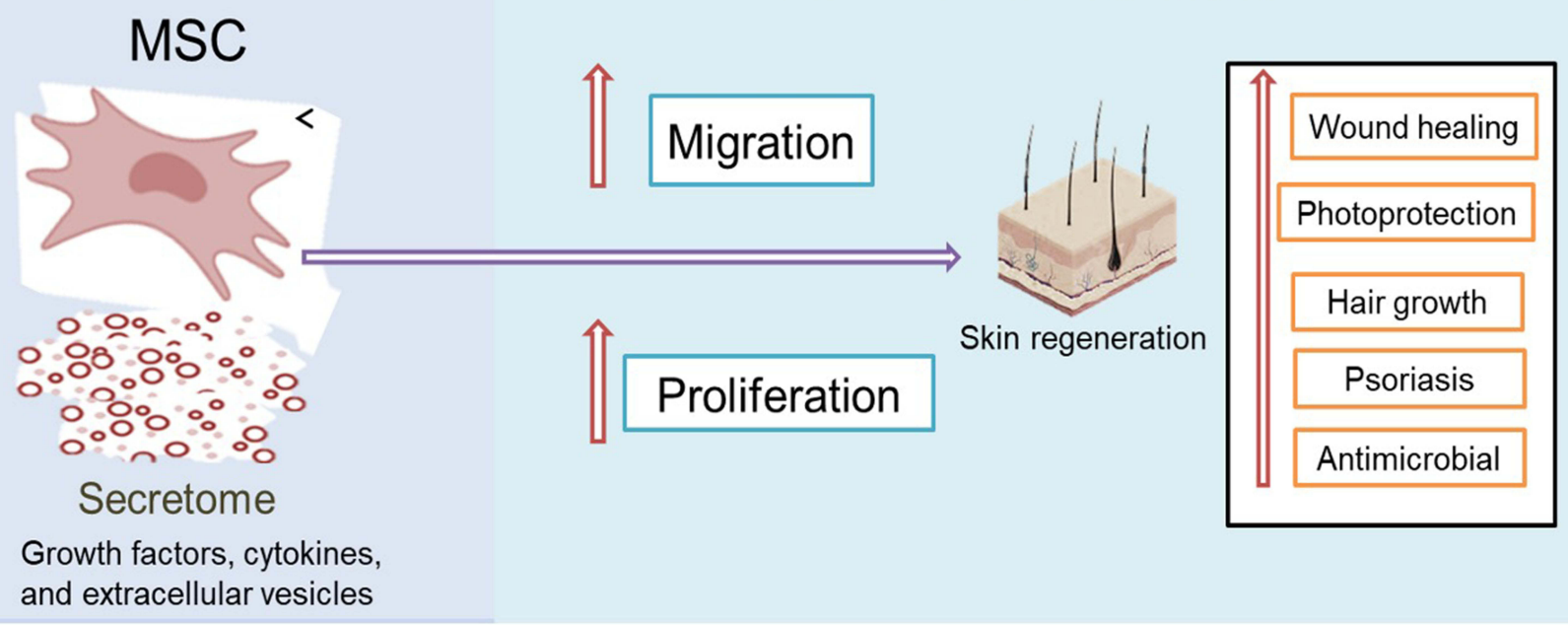

associated with the paracrine effect of important factors secreted by secretome. Because of awareness related to the work of MSCs based on the secretion of its secretome, it can be used as an alternative to overcome problems related to the use of living cells. ${ }^{8}$

Secretome is a bioactive molecule secreted by MSCs in conditioned media. It contains a large number of growth factors, cytokines, and various macromolecules and extracellular vesicles including microvesicles and exosomes that can stimulate various biological reactions, especially in modulation of various new tissue formations. ${ }^{8-12}$ These factors play an important role in communication between cells and are involved in a variety of physiological processes, including signal transduction to provide a biological response. ${ }^{13}$ Therapy using secretome can answer challenges related to the problem of living cell use. The secretome's ability to regenerate and repair damaged tissues makes it one of the alternatives related to the handling of various skin problems that have been widely reported in various studies. So, in this article, we will review the use of secretome for skin application is described in Table 1.

\section{Methodology}

This article is based on the results of the expansion of several sites, such as Scopus, PubMed, Science Direct, and
Google Scholar with the keywords "MSC secretome for skin," "secretome for skin," "secretome dermatology," 'secretome conditioned medium for skin,' "secretome conditioned medium for skin wound," "secretome conditioned medium for aging," "secretome conditioned medium for hair growth," and "secretome conditioned medium for psoriasis." A flowchart of the article collection described in Figure 1.

\section{Secretome}

\section{Isolation and Secretome Culture}

Isolation is the first step in obtaining a secretome. Secretome of MSCs can be isolated from various adult tissues, including the spinal cord, ${ }^{38}$ adipose tissue, ${ }^{39}$ skin tissue, ${ }^{16}$ peripheral blood, ${ }^{21}$ and neonatal tissue, such as Wharton jelly, ${ }^{40}$ umbilical cord, ${ }^{17}$ amniotic membrane, ${ }^{41}$ and placenta. ${ }^{42}$ The isolation process is done to obtain the network that is being used. After the isolation process, cell culture is carried out. Based on Park et al, cell cultures were carried out by incubating isolated cells for 5 hours at a temperature of $37^{\circ} \mathrm{C}$ in the growth medium using Dulbecco's Modified Eagle Medium (DMEM), which contains 10\% fetal bovine serum (FBS), to provide cell nutrition needs and 250 $\mathrm{U} / \mathrm{mL}$ collagenase type 1 . It was then filtered with 40 $\mu \mathrm{m}$ strainer cells and then planted in a growth medium 
Table I Secretome Test Objects for Skin Application

\begin{tabular}{|c|c|c|c|c|}
\hline No. & Biology Activity & Test Object & Testing & Ref \\
\hline \multirow[t]{14}{*}{ I. } & \multirow[t]{14}{*}{ Cutaneous wound } & Human foreskin fibroblasts (HFFs) & In vitro & {$[14]$} \\
\hline & & Human immortalised keratinocytes ( $\mathrm{HaCaTs}$ ) & In vitro & {$[4,5]$} \\
\hline & & $\begin{array}{l}\text { Human dermal microvascular endothelial cells } \\
\text { (HDMECs) }\end{array}$ & In vitro & {$[14]$} \\
\hline & & Human dermal fibroblasts (HDFs) & In vitro & {$[8,15]$} \\
\hline & & Human endothelial cell & In vitro & {$[8]$} \\
\hline & & Human umbilical vein endothelial cells (HUVECs) & In vitro & {$[16]$} \\
\hline & & L929 Fibroblast & In vitro & [5] \\
\hline & & Wistar rats & In vivo & {$[17,18]$} \\
\hline & & C57BL/6J mice & In vivo & [19] \\
\hline & & Lepr ${ }^{\mathrm{db} / \mathrm{db}}$ mice & In vivo & [20] \\
\hline & & Porcine models/ pigs & In vivo & {$[21]$} \\
\hline & & Rabbit ear & In vivo & {$[22]$} \\
\hline & & Female SCID Mice 8-9 weeks & In vivo & {$[4]$} \\
\hline & & Male volunteers & Clinic Phase I & [23] \\
\hline \multirow[t]{7}{*}{2.} & \multirow[t]{7}{*}{ Photoprotection } & Human dermal fibroblasts (HDFs) & In vitro & {$[24,25]$} \\
\hline & & Normal human dermal fibroblast (NHDF) & In vitro & {$[26]$} \\
\hline & & Human dermal fibroblast (HFF-I) & In vitro & {$[27]$} \\
\hline & & Human immortalized keratinocytes ( $\mathrm{HaCaTs}$ ) & In vitro & {$[28]$} \\
\hline & & NIH-3T3 cell & In vitro & [29] \\
\hline & & Nude mice & In vivo & {$[24,28]$} \\
\hline & & Human Skin & Clinical test & {$[30]$} \\
\hline \multirow[t]{6}{*}{3.} & \multirow[t]{6}{*}{ Hair Growth } & Human follicle dermal papilla cells (HFDPCs) & In vitro & [3I-33] \\
\hline & & Human epithelial keratinocytes (HEKs) & In vitro & {$[31]$} \\
\hline & & Outer root sheath cells (ORC) & In vitro & [34] \\
\hline & & $\mathrm{C} 3 \mathrm{H} / \mathrm{NeH}$ mice & In vivo & {$[31,34]$} \\
\hline & & C57BL/6 mice & In vivo & {$[33,35]$} \\
\hline & & Nude mice & In vivo & [32] \\
\hline 4. & Psoriasis & Wistar rats & In vivo & [36] \\
\hline 5 & $\begin{array}{l}\text { Other } \\
\text { (antimicrobial) }\end{array}$ & Rats & In vivo & [37] \\
\hline
\end{tabular}

(ie, basal growth of medium EBM-2 endothelial cells and equipped with EGM-2 supplements at $37^{\circ} \mathrm{C}$ and $5 \%$ $\left.\mathrm{CO}_{2}\right)^{2,38,43}$
Preparation of Secretome

Secretome preparation was performed when the cell reached $80-90 \%$ confluence, and it was then washed 


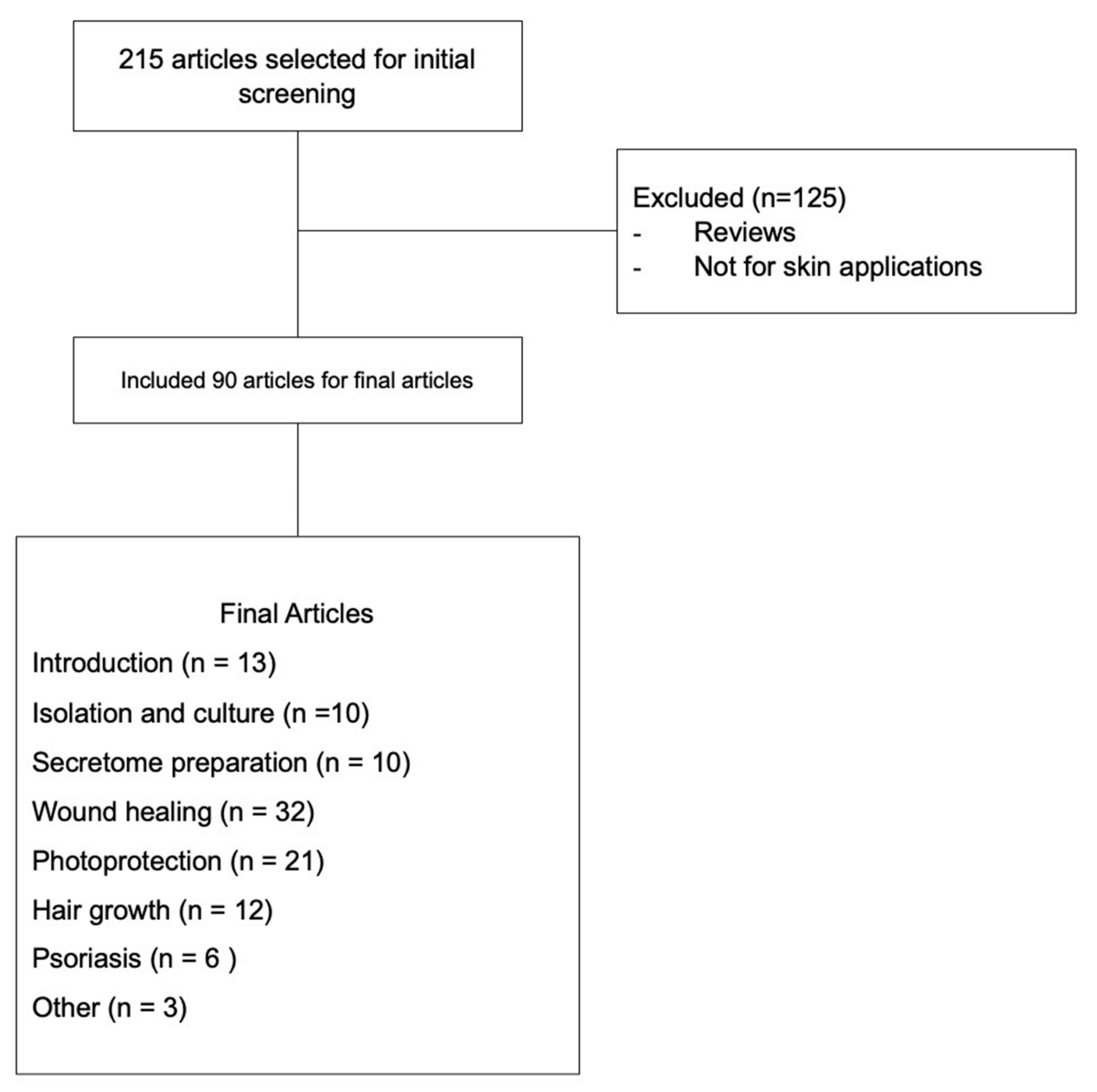

Figure I Flowchart of the article collection.

with PBS two times and incubated in DMEM (without FBS and antibiotics). Conditioned medium, or secretome, was collected after being incubated for 48 hours and centrifuged at $1500 \mathrm{rpm}$ for 3 minutes two times, then filtered with a syringe filter $0.45 \mu \mathrm{m}$ to remove debris and dead cells. The obtained conditioned medium was stored at $-80^{\circ} \mathrm{C}$ before use in testing. ${ }^{2,44}$

In some circumstances, due to the concentration of bioactive factors is low in the culture medium to produce a therapeutic effect, there are several methods were used to increase the secretion of these factors, namely culture in hypoxic conditions ${ }^{44,45}$ and planting in a two- (2D) or threedimensional (3D) matrix. ${ }^{17}$ Culturing MSCs in hypoxic in vitro conditions can increase the proliferation and migration effects of keratinocytes and dermal fibroblasts, which are related to the upregulation of angiogenesis factors, such as VEGF and bFGF, and increased collagen production compared to normoxia. ${ }^{45-47}$ Sun et al stated that protein levels in hypoxic cultures were 15 times greater than those in normal cultures. ${ }^{48}$
In 3D cultures, there was an increase in re-epithelialization related to success in wound closure as compared to normal cultures. $^{49}$

To identify the various proteins contained in the secretome, analysis techniques were required. Analysis techniques were performed to identify various proteins contained in the secretome. Conventional ELISA methods and the multiplex method were generally used. ${ }^{27}$ These methods can identify a number of proteins contained in the secretome, such as growth factors and cytokines. ${ }^{50,51}$ Research by Amirthalingam et al revealed that there are 28 protein analytes in the secretome of BM. The presence of growth factors, such as VEGF and TGF- $\beta$, in the secretome plays an important role in skin health. ${ }^{27}$

\section{The Role of Secretome on the Skin Application}

Secretome is known to have a variety of benefits in the treatment of various diseases. The main factor that plays 
a role is the secreted protein, which can help repair and regenerate damaged tissue. Secretome of MSCs plays an important role as a therapeutic agent in dealing with various skin problems. A list of potential effect of secretome in skin application can be seen in Table 2 .

\section{Wound Healing}

Wound healing is a natural process of the body that repairs and regenerates damaged tissues and consists of three main phases - inflammation, proliferation, and remodeling that occur in overlap. Wound healing is still a challenge in clinical management therapy problems, especially in chronic conditions. ${ }^{2}$ The use of secretome as an alternative therapy in dealing with skin injuries has been widely reported, including accelerating wound closure. ${ }^{42}$ Various studies to assess the benefits of secretome to wound healing have been done, both in vitro and in vivo. In vitro testing is generally done by looking at the migration and proliferation capabilities of cells. Use based on cell models has been tested using components of different types of skin cells, such as human immortalized keratinocytes (HaCaTs), ${ }^{14}$ human dermal fibroblasts (HDFs), ${ }^{15}$ human foreskin fibroblasts (HFFs) ${ }^{66}$ human dermal microvascular endothelial cells (HDMECs), ${ }^{14}$ and human umbilical vein endothelial cells (HUVECs). ${ }^{16}$ Cell migration capability can be determined using the assay migration method and/or starch assay. ${ }^{2}$ This test was conducted by comparing the speed of wound closure between control and treatment groups. To confirm the effects of cell migration, it can also be done by identifying the expression levels of MMP2 and MMP9, both of which are involved in the migration processes of cells. ${ }^{2}$ The proliferation ability of cells can be curated using the assay proliferation method, WST-1 assay and MTT assay. $^{2,14,15}$ This test aims to see if there is an increase in viability of the cells after secretome administration. Another supporting method can also be done by looking at the ability of angiogenesis using the angiogenesis assay. ${ }^{16}$

Secretome from a variety of different sources (such as bone marrow, adipose tissue, neonatal tissue, skin tissue and peripheral blood) have been shown to improve the migration ability of various dermal cells, such as fibroblasts, endothelial cells and keratinocytes, and the epidermis was able to stimulate the proliferation of these cells in vitro. ${ }^{19,67}$ Research by Miranda et al also stated that secretome from umbilical cord tissue-derived $\left(\mathrm{UCX}^{\circledR}\right)$ was able to increase HDF and HaCaT cell migration based on scratch test results. ${ }^{4}$ Sera et al also noted that there was an increase in expression of ki67 markers involved in cell proliferation by treatment using secretome with MTT assay method. ${ }^{2}$ The use of secretome was also able to modulate tube formation based on in vitro angiogenesis tests along with the migration and proliferation HUVECs. ${ }^{16,68-70}$ The speed of healing of wounds could be attributed to the trophic effects of MSCs, which affect dermal fibroblasts and keratinocytes. ${ }^{5}$ Interestingly, Topouzi et al used secretome from the dermal papilla fibroblast follicles for wound healing therapy and showed that the wound healing increased by 1.8 times faster than control. $^{57}$

In vivo experimental animal models can be used in the form of mice, ${ }^{53}$ rats, ${ }^{55}$ and pigs. ${ }^{21}$ This method has been tested on animal models with various conditions, such as chronic wounds, ${ }^{58}$ burns,${ }^{38}$ diabetic foot ulcers, ${ }^{71}$ skin ulcers, ${ }^{53}$ and radiation wounds, ${ }^{72}$ which require special handlers. The results showed that wound treatment with secretome was able to accelerate wound healing by increasing the effect of cell migration and proliferation and triggering angiogenesis, re-epithelialization, neovascularization and deposition of collagen that play a role in the wound healing process. ${ }^{53,73-75}$ According to Bari et al, secretome components in ADSC such as decorin (Dcn) and tenascin (Tnc) play an important role in epidermal growth factor receptor (EGFR) regulation. ${ }^{53}$

Various studies have been conducted to support data related to the benefits of secretome in vivo. Hackers et al studied the effects of secretome from peripheral blood mononuclear cells (SecPBMCs) and PBMC apoptosis (Apo-SecPBMC) on models of pigs with burns, showing an increase in the number of $\mathrm{CD} 31+$, which is especially greater in treatment with Apo-SecPBMCs, as well as a decrease in the number of mast cells in the wound area. ${ }^{21}$ Using the same secretome source, Mildner et al found that there was a significant increase in the number of $\mathrm{CD} 31+$ cells that play a role in the angiogenesis process in the SecPBMCs group. ${ }^{19}$ Similarly, Wagner et al revealed that the use of secretome from PBMCs is a promising therapy in improving wound healing that has been tested on Lepr ${ }^{\mathrm{db} / \mathrm{db}}$ mice. ${ }^{20}$ Secretome of adipose stromal stem cell (ASCs) in hypoxia showed a greater reduction in wound area compared to that in normoxia. ${ }^{47}$

The use of SecPBMCs has been further thoroughly related to toxicity testing using animal models of mice (assessing systemic toxicity) and mini pigs (local toxicity studies). The results showed that the minimum dose and 


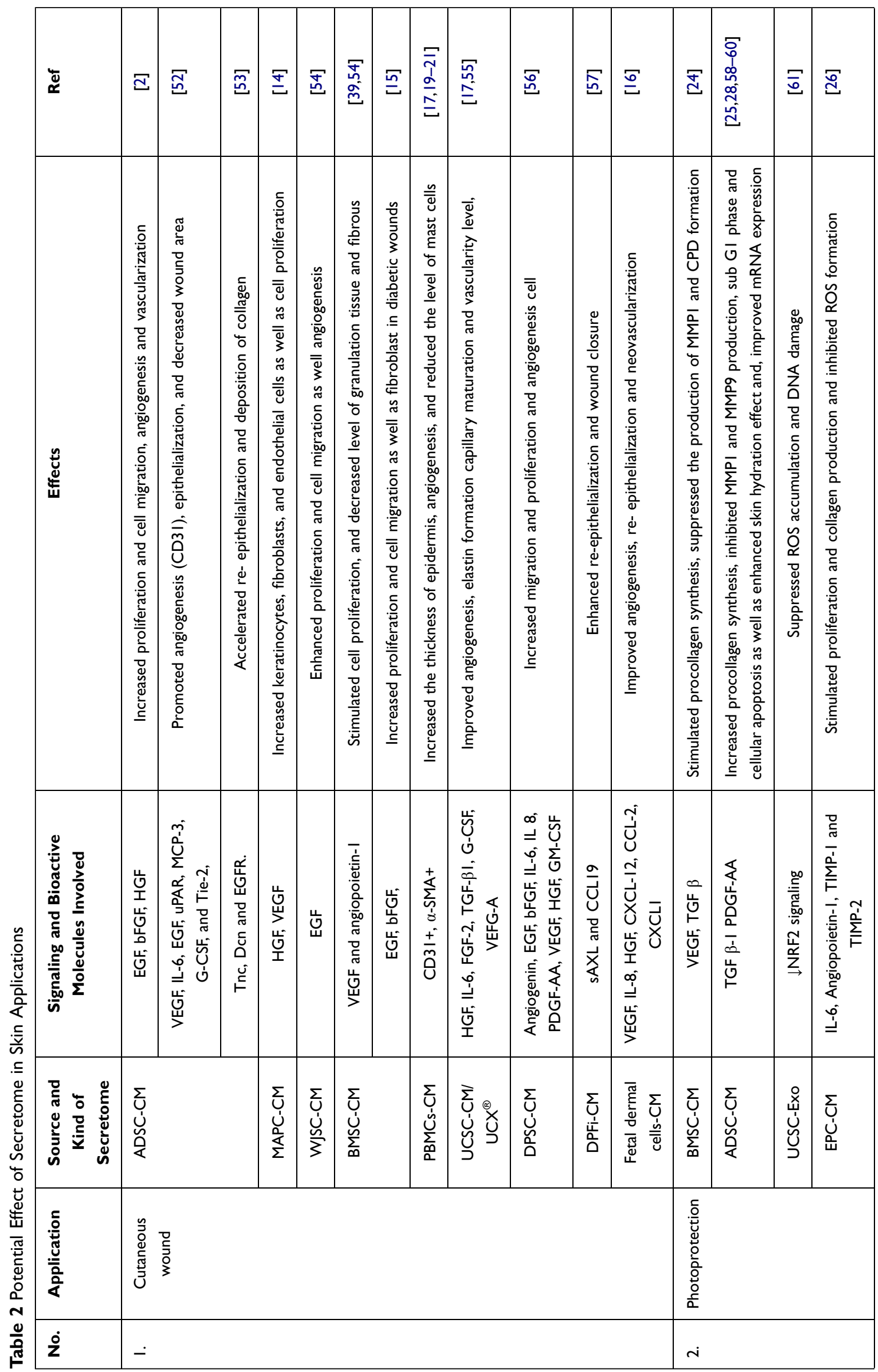




\begin{tabular}{|c|c|c|c|c|c|c|}
\hline$\overline{\bar{m}}$ & 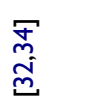 & $\stackrel{\bar{m}}{\tilde{c}}$ & $\widetilde{\widetilde{\sigma}}$ & 芯 & 壭 & గొ \\
\hline 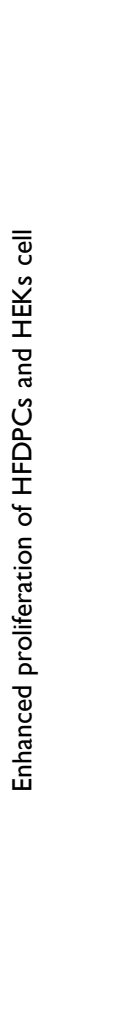 & 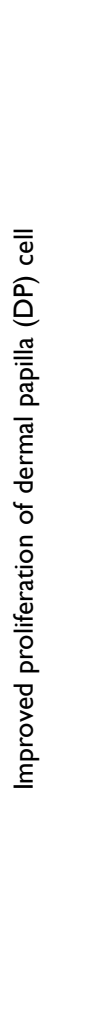 & 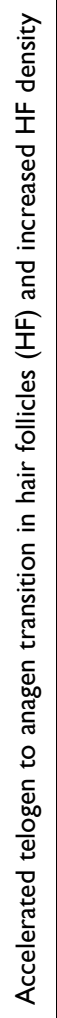 & 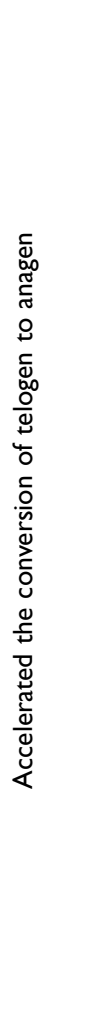 & 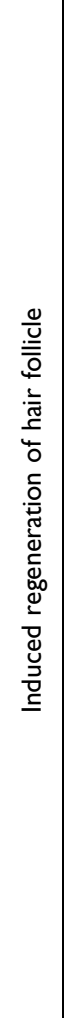 & 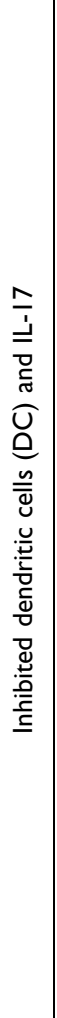 & 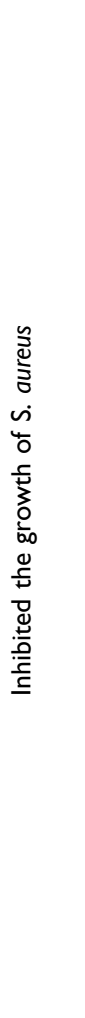 \\
\hline 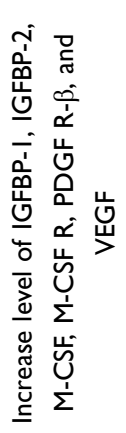 & 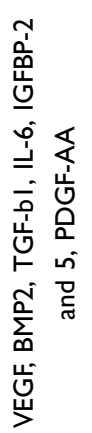 & 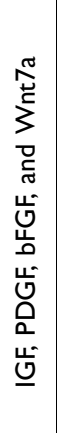 & 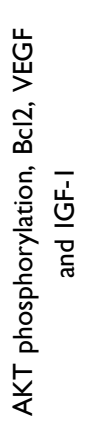 & 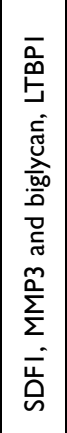 & 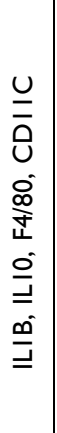 & 命 \\
\hline $\begin{array}{l}\sum_{u} \\
\text { Uू } \\
\stackrel{\alpha}{<}\end{array}$ & 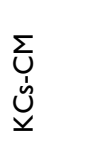 & 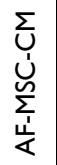 & $\begin{array}{l}\text { 岁 } \\
\sum_{\infty}^{n}\end{array}$ & $\mid \begin{array}{l}\Sigma \\
\text { D } \\
0 \\
0\end{array}$ & $\begin{array}{l}\frac{\mathscr{g}}{\varepsilon} \\
\frac{5}{\bar{v}}\end{array}$ & 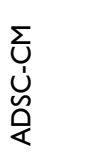 \\
\hline 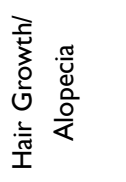 & & & & & 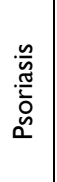 & 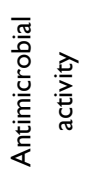 \\
\hline$\dot{m}$ & & & & & $\dot{+}$ & in \\
\hline
\end{tabular}

the maximum dose considered for the treatment of diabetic foot ulcers (DFUs) were $0.42 \mathrm{U} / \mathrm{kg}$ and $3.3 \mathrm{U} / \mathrm{kg}$, respectively. ${ }^{76}$

The results showed that the administration of secretome was able to accelerate the wound healing process compared to control. Secretome with a dose of $30 \mu \mathrm{g} / \mathrm{mL}$ of bone marrow (BMSC-CM) resulted in a maximum wound closure of $90 \%$ on the fifth day after treatment. ${ }^{2}$ While the secretome of the multipotent adult progenitor cell (MAPC-CM) used a dose of $100 \mu \mathrm{L}$ of $20 \times$ the concentration of conditioned medium to test wound healing in trial animals receiving intradermal injections. ${ }^{14}$ In clinical testing, phase 1 included secretome from PBMCs (Apo-SecPBMCs) for chronic wound treatment. This study was conducted to assess the safety of dosages used for topical applications, supernatant of $12.5 \times 10^{6}$ low dose PBMCs and equivalents of $25 \times 10^{6}$ PBMCs resuspended in NuGel Hydrogel high doses. Their results showed the use of Apo-SecPBMCs with low and high doses is safe and well-tolerated by the skin and has an effect on the reduction of the wound area. ${ }^{23}$

\section{Photoprotection}

Ultraviolet (UV) radiation can cause some skin problems, especially photoaging. Photoaging is skin aging caused by excessive UV exposure, which causes morphological and physiological changes of the skin such as the appearance of wrinkles and reduced skin elasticity. ${ }^{27}$ Research into the secretome as a therapy to prevent the influence of photoaging has been reported. Several in vitro models of skin cells, HDFs and HaCaTs, both of which are cells that function against defenses due to UVB radiation, were used. ${ }^{28}$ UV radiation can cause inhibition of the cell proliferation rate and reductions of collagen I, collagen III and elastin due to downregulation of expression of mRNA. ${ }^{58}$ UV radiation can also cause increased levels of MMP1 and MMP9, resulting in inhibited synthesis of procollagens and triggering activation of various signaling pathways, namely mitogen activated protein kinase (MAPK), protein activator 1 (AP-1) and nuclear factor kappa B (NF- $\mathrm{BB})$, which contributes to cell damage due to ROS formation and can trigger the apoptosis of skin cells, so as to inhibit skin regeneration and be able to inflict DNA damage in keratinocytes. $^{24,25,28,29,77}$

Various studies have been conducted to investigate the effects of secretome as an agent in the fight against photoaging. In vitro testing was done by various methods. Methods that were used looked at cell proliferation 
activity, cellular aging and cellular apoptosis, intracellular ROS levels, and pyrimidine cyclobutane dimer assay (CPD). ${ }^{26,27,29,58,78}$ Wang et al reported that there was an increase in cell proliferation from adipose-derived stem cells (ADSC-CM) use, as well as a decrease in the number of cells undergoing apoptosis and a decrease in cellular aging in HDFs. ${ }^{58} \mathrm{Kim}$ et al also found ADSC-CM can decrease the number of cell deaths from apoptosis (characterized by a decrease in the sub-G1 phase) and increase collagen I synthesis, as well as inhibit the production of MMP1 in HDFs. ${ }^{60}$ Using the same method of sub-GI assay, Dewi et al also reported a reduction in the percentage of apoptosis after treatment with the human umbilical cord blood mesenchymal stem cell (hUCB-MSC-CM) conformation $5 \%, 10 \%$ and $20 \%$ from $94.2 \%$ to $46.8 \%$, $31.8 \%$ and $31.5 \%$, respectively. ${ }^{29}$ The use of secretome was able to reduce intracellular ROS levels using the DCFDA fluorescent assay, ${ }^{26}$ as well as able to increase cell migration and reduce the level of formation of $\mathrm{CPD}$ in vitro. $^{27}$

The secretome of ADSCs had the effect of providing photoprotection. This is related to the content of biological factors that play a role in particular platelet-derived growth factor AA (PDGF-AA), which can promote deposition and remodeling of the extracellular matrix. ${ }^{25}$ This effect is also associated with the content of TGF- $\beta 1$ in the secretome, which can stimulate mRNA expression and increase collagen production. ${ }^{59}$ This mechanism is also related to the upregulated expression of wnt3a and catenin in the Wnt/ $\beta$ catenin signaling pathway, which is associated with increased expression of TGF- $\beta 2$ (which is important for the synthesis of procollagen type I). ${ }^{79}$

Other tests performed on the test animals, which were generally nude mice, pertained to UVB exposure. The results revealed a reduction in wrinkles and skin distress by improving skin hydration after macroscopic use of secretome and increased collagen synthesis based on Masson trichrome analysis. ${ }^{24,27,28}$

In some studies, it has been reported that secretome can be used as an active ingredient in cosmetics, reminding us of its benefits in regenerating the skin. Generally, research utilizes secretome as a skin care therapy to prevent various aging factors, including photoaging. Amirthalingam et al formulated secretome as an antiaging cosmetic product in the form of semi-solid serum preparations, with the following doses used $0.25 \%, 0.5 \%$ and $1 \%{ }^{27}$ Similar research by Kim et al utilized secretome as raw material in the manufacture of cosmetics applied using an air brush, with secretome concentration as high as $5 \%$. The results showed a reduction in test parameters, such as wrinkles, and increased skin moisture. ${ }^{80}$ Similarly, Kim et al also used secretome as a raw material cosmetic material in the form of cream preparations. The secretome concentration used was $1 \%$, which was contoured in $3 \mathrm{D}$ culture. The use of secretome can increase collagen synthesis greater than control and reduced aging by improving skin elasticity. ${ }^{30}$

\section{Hair Growth}

Alopecia is a term used to state the condition of baldness or hair loss due to abnormalities in the scalp that can be caused by various factors. ${ }^{33}$ This condition causes the active phase of hair growth (anagen stage) to be inhibited, while the rest phase (telogen stage) becomes faster in the hair growth cycle. ${ }^{35,81}$ So, a lot of research focuses on understanding the cycle of hair growth. The effect of secretome therapy on hair growth has been reported in various studies. In vitro testing is generally conducted to determine the proliferation ability of hair cells. Used human papilla cells of human follicles (HFDPCs), outer root sheath cells (ORCs) and human epithelial keratinocytes (HEKs), ${ }^{31,34,82}$ which are types of cells located in hair follicles that can stimulate hair growth and regeneration through reciprocal communication processes with epithelial cells. $^{31-33}$

Zhang et al investigated the paracrine factors that may be involved in hair follicle regeneration using secretome from dermal papilla cells (DPCs). The results showed that the use of the secretome of DPCs in passage 3 secreted a large amount of CXCL12, MMP3 and biglycan, which played a role in the activation of the $\mathrm{Wnt} / \beta$-catenin signaling line, as well as LTBP1. ${ }^{63}$ Activation of this pathway is known to trigger the proliferation of hair follicle cells. $^{83,84}$ The use of secretome is able to trigger the proliferation of HFDPCs and ORCs, to accelerate the telogen phase to anagen and ex vivo can induce proliferation of the hair matrix. In the application using animal models in the form of mice $\mathrm{C} 3 \mathrm{H} / \mathrm{HeN}$, secretome was able to induce hair follicle growth. ${ }^{34} \mathrm{Pu}$ et al also reported that secretome was able to trigger proliferation and increase hair follicle growth in mice ischemia/reperfusion- (I/R-) models. $^{85}$

\section{Psoriasis}

Psoriasis is a chronic inflammatory condition of the skin that causes increased levels of expression of interleukin 
(IL-17). In psoriasis, Langerhans cells represent a disorder in the migration of epidermis cells that serve as an immune response associated with $\mathrm{T}$ cell responses, particularly Th17-mediated. ${ }^{86}$ This condition also causes abnormalities in cytokine production that can cause epidermis hyperplasia as well as abnormal keratinocyte apoptosis (KCs). ${ }^{87}$ Psoriasis is characterized by the appearance of patches or rashes with thick white scales on the skin and nails. ${ }^{88}$

Studies on secretome in the treatment of psoriasis have been conducted. Psoriasis modeling can be uses using imiquimod with Wistar rats models. Imiquimod causes inflammation characterized by the presence of coarse lamela and excoriation. The use of secretome is able to reduce the effects of inflammation caused by the use of imiquimod (IMQ) better than control. ${ }^{36}$ Zhang et al stated that exosomes (secretome) is able to decrease psoriasis score in IMQ rats model through inhibiting of maturation and activation of dendritic cells (DC) and IL-17 in HaCaTs. ${ }^{89}$ Seetharaman et al reported that the secretome of adipose tissue, which is administered topically, showed a significant decrease in the amount of erythematous plaque and silver scales on the scalp of the sufferer after administration of the secretome for 2 weeks and disappeared after one month of administration. ${ }^{88}$

\section{Other Application}

Secretome is reported to have antimicrobial activity. Antimicrobial is a compound that can inhibit or suppress the growth of microbes. Various drugs that are resistant to microbes continue to increase. The use of secretome can be one of the alternatives to prevent drug resistance. In some studies, secretome is known to release antimicrobial peptides such as cathelicidin LL-37, calprotectin, hepcidin, RNase3, lipocalin-2 and human $\beta$-defensins. ${ }^{37}$ Yagi et al reported secretome of adipose tissue is able to suppress the growth of S. aureus through increased activity of cathelicidin. ${ }^{65}$ Harman et al investigated the antibacterial effects of secretome on bacteria found in skin wounds, tested using S. aureus and E. coli. The results showed that the use of secretome can inhibit the growth of gram-positive bacteria S. aureus and gram negative E. coli from the production of secreted factors that can affect bacterial membranes. ${ }^{90}$

\section{Conclusion}

Secretome, a bioactive factor secreted by MSCs, has a variety of benefits on the skin as a therapeutic agent for various regenerative diseases. Research on the use of secretome for skin applications and formulation development are still very limited. Several studies reported that the process to obtain secretome from MSCs was through isolation from various adult tissues and cultured in a medium of growth. The culture process can affect the level of expression of the resulting factors. Applications of the secretome for skin include wound healing, photoprotection, promotion of hair growth, psoriasis treatment, and other application as antimicrobial. Considering the various constituents of secretome, it has a lot of potential in various diseases needing the development of and more indepth studies in order to be maximally used.

\section{Abbreviations}

aSMA, smooth muscle cell-specific alpha actin; ADSC, adipose-derived stem cell; AF-MSC, amniotic fluidderived mesenchymal stem cell; Bcl-2; B-cell lymphoma 2; bFGF, basic fibroblast growth factor; BMSC, bone marrow stem cell; $\mathrm{CD}$, cluster of differentiation; $\mathrm{CM}$, Conditioned medium; Dcn, decorin; DPFi, dermal papilla fibroblasts; DPSC, dental pulp stem cell; EGF, epidermal growth factor; EGFR, epidermal growth factor receptor; EPC, endothelial progenitor cells; EV, extracellular vesicle; Exo, Exosome; G-CSF, granulocyte-colony stimulating factor; GM-CSF, granulocyte-macrophage colony-stimulating factor; HGF, hepatocyte growth factor; IGF, insulin-like growth factor; IGFBP, insulin-like growth factor binding protein; IL, Interleukin; KCs, interfollicular keratinocytes; LTBP1, latent TGFbeta binding protein 1; MAPC, multipotent adult progenitor cell; M-CSF, Macrophage-colony stimulating factor; MMP, matrix metalloproteinase; MSC, mesenchymal stem cell; PBS, phosphate buffered saline; PBMC, peripheral blood mononuclear cells; PDGF, platelet-derived growth factor; ROS, reactive oxygen species; SDF1, stromal cell-derived factor 1 ; TGF- $\beta$, transforming growth factor- $\beta$; TIMP-1, tissue inhibitor metalloproteinase 1; Tnc, tenascin; UV, ultraviolet; UCSC, umbilical cord stem cells; VEGF, vascular endothelial growth factor; WJSC, wharton's jelly stem cells.

\section{Acknowledgment}

The authors thank Dr. apt. Bayu Winata Putera, M.Si and Dr Cosmos Mangunsong Sp.M(K) for supporting the secretome research in Universitas Padjadjaran.

\section{Disclosure}

The authors report no conflicts of interest in this work. 


\section{References}

1. Robert AW, Azevedo Gomes F, Rode MP, et al. The skin regeneration potential of a pro-angiogenic secretome from human skin-derived multipotent stromal cells. $J$ Tissue Eng. 2019;10:2041731419833391. doi:10.1177/2041731419833391

2. Park S-R, Kim J-W, Jun H-S, Roh JY, Lee H-Y, Hong I-S. Stem cell secretome and its effect on cellular mechanisms relevant to wound healing. Mol Therapy. 2018;26(2):606-617. doi:10.1016/j. ymthe.2017.09.023

3. Linero I, Chaparro O. Paracrine effect of mesenchymal stem cells derived from human adipose tissue in bone regeneration. PLoS One. 2014;9(9):e107001. doi:10.1371/journal.pone.0107001

4. Miranda JP, Filipe E, Fernandes AS, et al. The human umbilical cord tissue-derived MSC population $\mathrm{UCX}^{\circledR}$ promotes early motogenic effects on keratinocytes and fibroblasts and G-CSF-mediated mobilization of BM-MSCs when transplanted in vivo. Cell Transplant. 2015;24(5):865-877.

5. Walter MN, Wright KT, Fuller HR, MacNeil S, Johnson WEB. Mesenchymal stem cell-conditioned medium accelerates skin wound healing: an in vitro study of fibroblast and keratinocyte scratch assays. Exp Cell Res. 2010;316(7):1271-1281.

6. Bhang SH, Lee S, Shin J-Y, Lee T-J, Jang H-K, Kim B-S. Efficacious and clinically relevant conditioned medium of human adipose-derived stem cells for therapeutic angiogenesis. Mol Therapy. 2014;22(4):862-872.

7. Rubio D, Garcia S, De la Cueva T, et al. Human mesenchymal stem cell transformation is associated with a mesenchymal-epithelial transition. Exp Cell Res. 2008;314(4):691-698.

8. Sagaradze G, Grigorieva O, Nimiritsky P, et al. Conditioned medium from human mesenchymal stromal cells: towards the clinical translation. Int J Mol Sci. 2019;20(7):1656.

9. Balasubramanian S, Thej C, Walvekar A, et al. Evaluation of the secretome profile and functional characteristics of human bone marrow mesenchymal stromal cells-derived conditioned medium suggest potential for skin rejuvenation. $J$ Cosmetics Dermatol Sci Applications. 2017;7(01):99.

10. Bellei B, Migliano E, Tedesco M, et al. Adipose tissue-derived extracellular fraction characterization: biological and clinical considerations in regenerative medicine. Stem Cell Res Ther. 2018;9 (1):1-18. doi:10.1186/s13287-018-0956-4

11. Lopatina T, Bruno S, Tetta C, Kalinina N, Porta M, Camussi G. Platelet-derived growth factor regulates the secretion of extracellular vesicles by adipose mesenchymal stem cells and enhances their angiogenic potential. Cell Commun Signaling. 2014;12(1):1-12. doi:10.1186/1478-811X-12-26

12. Niada S, Giannasi C, Magagnotti C, Andolfo A, Brini AT. Proteomic analysis of extracellular vesicles and conditioned medium from human adipose-derived stem/stromal cells and dermal fibroblasts. J Proteomics. 2021;232:104069.

13. Wei W, Riley NM, Yang AC, et al. Cell type-selective secretome profiling in vivo. Nat Chem Biol. 2021;17(3):326-334. doi:10.1038/ s41589-020-00698-y

14. Ahangar P, Mills SJ, Smith LE, et al. Human multipotent adult progenitor cell-conditioned medium improves wound healing through modulating inflammation and angiogenesis in mice. Stem Cell Res Ther. 2020;11(1):1-16. doi:10.1186/s13287-020-01819-z

15. Saheli M, Bayat M, Ganji R, et al. Human mesenchymal stem cells-conditioned medium improves diabetic wound healing mainly through modulating fibroblast behaviors. Arch Dermatol Res. 2020;312(5):325-336. doi:10.1007/s00403-019-02016-6

16. Chinnici CM, Amico G, Gallo A, et al. Small Extracellular Vesicles from Human Fetal Dermal Cells and Their MicroRNA Cargo: KEGG Signaling Pathways Associated with Angiogenesis and Wound Healing. Stem Cells Int. 2020;2020:1-18. doi:10.1155/2020/8889379
17. Santos JM, Camões SP, Filipe E, et al. Three-dimensional spheroid cell culture of umbilical cord tissue-derived mesenchymal stromal cells leads to enhanced paracrine induction of wound healing. Stem Cell Res Ther. 2015;6(1):1-19. doi:10.1186/s13287-015-0082-5

18. Pouriran R, Piryaei A, Mostafavinia A, et al. The effect of combined pulsed wave low-level laser therapy and human bone marrow mesenchymal stem cell-conditioned medium on open skin wound healing in diabetic rats. Photomed Laser Surg. 2016;34(8):345-354. doi:10.1089/pho.2015.4020

19. Mildner M, Hacker S, Haider T, et al. Secretome of peripheral blood mononuclear cells enhances wound healing. PLoS One. 2013;8(3): e60103. doi:10.1371/journal.pone. 0060103

20. Wagner T, Traxler D, Simader E, et al. Different pro-angiogenic potential of $\gamma$-irradiated PBMC-derived secretome and its subfractions. Sci Rep. 2018;8(1):1-15. doi:10.1038/s41598-018-36928-6

21. Hacker S, Mittermayr R, Nickl S, et al. Paracrine factors from irradiated peripheral blood mononuclear cells improve skin regeneration and angiogenesis in a porcine burn model. Sci Rep. 2016;6 (1):1-13. doi:10.1038/srep25168

22. Hu C-H, Tseng Y-W, Chiou C-Y, et al. Bone marrow concentrate-induced mesenchymal stem cell conditioned medium facilitates wound healing and prevents hypertrophic scar formation in a rabbit ear model. Stem Cell Res Ther. 2019;10(1):1-13.

23. Simader E, Traxler D, Kasiri MM, et al. Safety and tolerability of topically administered autologous, apoptotic PBMC secretome (APOSEC) in dermal wounds: a randomized Phase 1 trial (MARSYAS I). Sci Rep. 2017;7 (1):1-8. doi:10.1038/s41598-017-06223-x

24. Kwon TR, Oh CT, Choi EJ, et al. Conditioned medium from human bone marrow-derived mesenchymal stem cells promotes skin moisturization and effacement of wrinkles in UVB-irradiated SKH-1 hairless mice. Photodermatol Photoimmunol Photomed. 2016;32 (3):120-128. doi:10.1111/phpp.12224

25. Guo S, Wang $\mathrm{T}$, Zhang $\mathrm{S}$, et al. Adipose-derived stem cell-conditioned medium protects fibroblasts at different senescent degrees from UVB irradiation damages. Mol Cell Biochem. 2020;463(1):67-78. doi:10.1007/s11010-019-03630-8

26. Sohn SJ, Yu JM, Lee EY, et al. Anti-aging properties of conditioned media of epidermal progenitor cells derived from mesenchymal stem cells. Dermatol Ther (Heidelb). 2018;8(2):229-244. doi:10.1007/ s13555-018-0229-2

27. Amirthalingam M, Bhat S, Dighe $P$, Seetharam R. HumanMesenchymal-Stromal-Cells-Derived-Conditioned-Medium-BasedFormulation-for-Advanced-Skin-Care. Int J Mol. 2019;2:5.

28. Li L, Ngo HTT, Hwang E, et al. Conditioned Medium from Human Adipose-Derived Mesenchymal Stem Cell Culture Prevents UVB-Induced Skin Aging in Human Keratinocytes and Dermal Fibroblasts. Int J Mol Sci. 2019;21(1):49. doi:10.3390/ijms21010049

29. Dewi DAR, Sandra F. Conditioned Media of Human Umbilical Cord Blood Mesenchymal Stem Cell Inhibits Ultraviolet B-induced Apoptosis in Fibroblasts. Indonesian Biomed J. 2019;11(1):85-90. doi:10.18585/inabj.v11i1.544

30. Kim KH, Kim Y-S, Lee S, An S. The effect of three-dimensional cultured adipose tissue-derived mesenchymal stem cell-conditioned medium and the antiaging effect of cosmetic products containing the medium. Biomed Dermatol. 2020;4(1):1-12. doi:10.1186/s41702-019-0053-Z

31. Park B-S, Kim W-S, Choi J-S, et al. Hair growth stimulated by conditioned medium of adipose-derived stem cells is enhanced by hypoxia: evidence of increased growth factor secretion. Biomed Res. 2010;31(1):27-34. doi:10.2220/biomedres.31.27

32. Abreu CM, Cerqueira MT, Pirraco RP, Gasperini L, Reis RL, Marques AP. Rescuing key native traits in cultured dermal papilla cells for human hair regeneration. $J$ Adv Res. 2020.

33. Park J, Jun EK, Son D, et al. Overexpression of Nanog in amniotic fluid-derived mesenchymal stem cells accelerates dermal papilla cell activity and promotes hair follicle regeneration. Exp Mol Med. 2019;51(7):1-15. 
34. Won CH, Jeong Y-M, Kang S, et al. Hair-growth-promoting effect of conditioned medium of high integrin $\alpha 6$ and low CD 71 (a6bri/ CD71dim) positive keratinocyte cells. Int J Mol Sci. 2015;16 (3):4379-4391.

35. Hu S, Li Z, Lutz H, et al. Dermal exosomes containing miR-218-5p promote hair regeneration by regulating $\beta$-catenin signaling. Sci $A d v$. 2020;6(30):eaba1685.

36. Sarycheva MV, Nadezhdina NA, Nadezhdin SV, et al. Effect of multipotent mesenchymal stromal cells secretome on imiquimod-induced psoriasis in rats. $J$ Int Pharmaceutical Res. 2019;46(4):296-301.

37. Kasiri MM, Beer L, Nemec L, et al. Dying blood mononuclear cell secretome exerts antimicrobial activity. Eur J Clin Invest. 2016;46 (10):853-863.

38. Aryan A, Bayat M, Bonakdar S, et al. Human bone marrow mesenchymal stem cell conditioned medium promotes wound healing in deep second-degree burns in male rats. Cells Tissues Organs. 2018;206 (6):317-329.

39. Kober J, Gugerell A, Schmid M, et al. Wound healing effect of conditioned media obtained from adipose tissue on human skin cells: a comparative in vitro study. Ann Plast Surg. 2016;77(2):156-163.

40. Sun J, Zhang Y, Song X, Zhu J, Zhu Q. The healing effects of conditioned medium derived from mesenchymal stem cells on radiation-induced skin wounds in rats. Cell Transplant. 2019;28 (1):105-115.

41. Pan C, Lang H, Zhang T, et al. Conditioned medium derived from human amniotic stem cells delays $\mathrm{H} 2 \mathrm{O} 2$-induced premature senescence in human dermal fibroblasts. Int J Mol Med. 2019;44 (5):1629-1640.

42. Du L, Lv R, Yang X, Cheng S, Ma T, Xu J. Hypoxic conditioned medium of placenta-derived mesenchymal stem cells protects against scar formation. Life Sci. 2016;149:51-57.

43. Li M, Zhao Y, Hao H, et al. Umbilical cord-derived mesenchymal stromal cell-conditioned medium exerts in vitro antiaging effects in human fibroblasts. Cytotherapy. 2017;19(3):371-383.

44. Wiśniewska J, Słyszewska M, Stałanowska K, et al. Effect of PigAdipose-Derived Stem Cells' Conditioned Media on Skin WoundHealing Characteristics In Vitro. Int J Mol Sci. 2021;22(11):5469.

45. Hsiao ST, Lokmic Z, Peshavariya H, et al. Hypoxic conditioning enhances the angiogenic paracrine activity of human adipose-derived stem cells. Stem Cells Dev. 2013;22(10):1614-1623.

46. Kosol W, Kumar S, Marrero-BerrÍos I, Berthiaume F. Medium conditioned by human mesenchymal stromal cells reverses low serum and hypoxia-induced inhibition of wound closure. Biochem Biophys Res Commun. 2020;522(2):335-341.

47. Lee EY, Xia Y, Kim WS, et al. Hypoxia-enhanced wound-healing function of adipose-derived stem cells: increase in stem cell proliferation and up-regulation of VEGF and bFGF. Wound Repair Regeneration. 2009;17(4):540-547.

48. Sun B, Guo S, Xu F, et al. Concentrated hypoxia-preconditioned adipose mesenchymal stem cell-conditioned medium improves wounds healing in full-thickness skin defect model. Int Scholarly Res Notices. 2014;2014:458.

49. Collawn SS, Mobley JA, Banerjee NS, Chow LT. Conditioned media from adipose-derived stromal cells accelerates healing in 3-dimensional skin cultures. Ann Plast Surg. 2016;76(4):446-452.

50. Kuljanin M, Elgamal RM, Bell GI, et al. Quantitative proteomics evaluation of human multipotent stromal cell for $\beta$ cell regeneration. Cell Rep. 2018;25(9):2524-2536. e2524.

51. Wangler S, Kamali A, Wapp C, et al. Uncovering the secretome of mesenchymal stromal cells exposed to healthy, traumatic, and degenerative intervertebral discs: a proteomic analysis. Stem Cell Res Ther. 2021;12(1):1-17.

52. Luo Y, Yi X, Liang T, et al. Autograft microskin combined with adipose-derived stem cell enhances wound healing in a full-thickness skin defect mouse model. Stem Cell Res Ther. 2019;10(1):1-15.
53. Bari E, Di Silvestre D, Mastracci L, et al. GMP-compliant sponge-like dressing containing MSC lyo-secretome: proteomic network of healing in a murine wound model. Eur $J$ Pharmaceutics Biopharmaceutics. 2020;155:37-48.

54. Payushina O, Butorina N, Sheveleva O, Domaratskaya E. Effect of mesenchymal stromal cells and conditioned media on healing of skin wound. Bull Exp Biol Med. 2018;165(4):572-575.

55. Mathen C, Sawant MG, Gupta R, Dsouza W, Krishna SG. Evaluation of Potential Application of Wharton's Jelly-Derived Human Mesenchymal Stromal Cells and its Conditioned Media for Dermal Regeneration using Rat Wound Healing Model. Cells Tissues Organs. 2021:1:1-14.

56. Jain RK, Vakil D, Cunningham C, Sidhu K. Human mesenchymal stem cells conditioned media promotes the wound healing process-An in vitro study. Stem Cell Res Ther. 2019.

57. Topouzi H, Boyle CJ, Williams G, Higgins CA. Harnessing the secretome of hair follicle fibroblasts to accelerate ex vivo healing of human skin wounds. J Investigative Dermatol. 2020;140(5):1075-1084. e1011.

58. Wang T, Guo S, Liu X, Xv N, Zhang S. Protective effects of adipose-derived stem cells secretome on human dermal fibroblasts from ageing damages. Int J Clin Exp Pathol. 2015;8(12):15739.

59. Cho J-W, Kang M-C, Lee K-S. TGF- $\beta 1$-treated ADSCs-CM promotes expression of type I collagen and MMP-1, migration of human skin fibroblasts, and wound healing in vitro and in vivo. Int J Mol Med. 2010;26(6):901-906.

60. Kim W-S, Park B-S, Park S-H, Kim H-K, Sung J-H. Antiwrinkle effect of adipose-derived stem cell: activation of dermal fibroblast by secretory factors. J Dermatol Sci. 2009;53(2):96-102.

61. Wang T, Jian Z, Baskys A, et al. MSC-derived exosomes protect against oxidative stress-induced skin injury via adaptive regulation of the NRF2 defense system. Biomaterials. 2020;257:120264.

62. Rajendran RL, Gangadaran P, Bak SS, et al. Extracellular vesicles derived from MSCs activates dermal papilla cell in vitro and promotes hair follicle conversion from telogen to anagen in mice. Sci Rep. 2017;7(1):1-12.

63. Zhang H, Zhu N-X, Huang K, et al. iTRAQ-based quantitative proteomic comparison of early-and late-passage human dermal papilla cell secretome in relation to inducing hair follicle regeneration. PLoS One. 2016;11(12):e0167474.

64. Evans EA, Sayers SR, Kodji X, et al. Psoriatic skin inflammation induces a pre-diabetic phenotype via the endocrine actions of skin secretome. Mol Metab. 2020;41:101047.

65. Yagi H, Chen AF, Hirsch D, et al. Antimicrobial activity of mesenchymal stem cells against Staphylococcus aureus. Stem Cell Res Ther. 2020;11(1):1-12.

66. Hu Q, Luni C, Elvassore N. Microfluidics for secretome analysis under enhanced endogenous signaling. Biochem Biophys Res Coтmun. 2018;497(2):480-484.

67. Li M, Luan F, Zhao Y, et al. Mesenchymal stem cell-conditioned medium accelerates wound healing with fewer scars. Int Wound $J$. 2017;14(1):64-73

68. Oskowitz A, McFerrin H, Gutschow M, Carter ML, Pochampally R. Serum-deprived human multipotent mesenchymal stromal cells (MSCs) are highly angiogenic. Stem Cell Res. 2011;6(3):215-225.

69. Rong X, Li J, Yang Y, Shi L, Jiang T. Human fetal skin-derived stem cell secretome enhances radiation-induced skin injury therapeutic effects by promoting angiogenesis. Stem Cell Res Ther. 2019;10 (1):1-11.

70. Chinnici CM, Iannolo G, Cittadini E, et al. Extracellular VesicleDerived microRNAs of Human Wharton's Jelly Mesenchymal Stromal Cells May Activate Endogenous VEGF-A to Promote Angiogenesis. Int J Mol Sci. 2021;22(4):2045.

71. De Gregorio C, Contador D, Díaz D, et al. Human adipose-derived mesenchymal stem cell-conditioned medium ameliorates polyneuropathy and foot ulceration in diabetic BKS $\mathrm{db} / \mathrm{db}$ mice. Stem Cell Res Ther. 2020;11:1-21. 
72. Brunchukov V, Astrelina T, Usupzhanova D, et al. Evaluation of the Effectiveness of Mesenchymal Stem Cells of the Placenta and Their Conditioned Medium in Local Radiation Injuries. Cells. 2020;9 (12):2558.

73. Mehanna RA, Nabil I, Attia N, et al. The effect of bone marrow-derived mesenchymal stem cells and their conditioned media topically delivered in fibrin glue on chronic wound healing in rats. Biomed Res Int. 2015;2015:584.

74. Gaetani M, Chinnici CM, Carreca AP, Di Pasquale C, Amico G, Conaldi PG. Unbiased and quantitative proteomics reveals highly increased angiogenesis induction by the secretome of mesenchymal stromal cells isolated from fetal rather than adult skin. J Tissue Eng Regen Med. 2018;12(2):e949-e961.

75. Chen L, Cheng L, Wang Z, et al. Conditioned medium-electrospun fiber biomaterials for skin regeneration. Bioactive Materials. 2021;6 (2):361-374.

76. Wuschko S, Gugerell A, Chabicovsky M, et al. Toxicological testing of allogeneic secretome derived from peripheral mononuclear cells (APOSEC): a novel cell-free therapeutic agent in skin disease. Sci Rep. 2019;9(1):1-14.

77. Dorr M, Guignard R, Auger F, Rochette P. The use of tissue-engineered skin to demonstrate the negative effect of CXCL5 on epidermal UV radiation-induced CPD repair efficiency. $\mathrm{Br}$ J Dermatol. 2020.

78. Song SY, Jung JE, Jeon YR, Tark KC, Lew DH. Determination of adipose-derived stem cell application on photo-aged fibroblasts, based on paracrine function. Cytotherapy. 2011;13(3):378-384.

79. Xu X, Wang H-Y, Zhang Y, et al. Adipose-derived stem cells cooperate with fractional carbon dioxide laser in antagonizing photoaging: a potential role of Wnt and $\beta$-catenin signaling. Cell Biosci. 2014;4 (1):1-11.

80. Kim HJ, Jung MS, Hur YK, Jung AH. A study on clinical effectiveness of cosmetics containing human stem cell conditioned media. Biomed Dermatol. 2020;4(1):1-11.

81. Yang G, Chen Q, Wen D, et al. A therapeutic microneedle patch made from hair-derived keratin for promoting hair regrowth. Acs Nano. 2019;13(4):4354-4360.
82. Jeong Y-M, Sung YK, Kim W-K, et al. Ultraviolet B preconditioning enhances the hair growth-promoting effects of adipose-derived stem cells via generation of reactive oxygen species. Stem Cells Dev. 2013;22(1):158-168.

83. Park P-J, Moon B-S, Lee S-H, et al. Hair growth-promoting effect of Aconiti Ciliare Tuber extract mediated by the activation of $\mathrm{Wnt} / \beta$ catenin signaling. Life Sci. 2012;91(19-20):935-943.

84. Myung PS, Takeo M, Ito M, Atit RP. Epithelial Wnt ligand secretion is required for adult hair follicle growth and regeneration. J Investigative Dermatol. 2013;133(1):31-41.

85. $\mathrm{Pu} \mathrm{C}-\mathrm{M}$, Chen $\mathrm{Y}-\mathrm{C}$, Chen $\mathrm{Y}-\mathrm{C}$, et al. Interleukin-6 from adipose-derived stem cells promotes tissue repair by the increase of cell proliferation and hair follicles in ischemia/reperfusion-treated skin flaps. Mediators Inflamm. 2019;2:2019.

86. Eaton L, Mellody K, Pilkington S, Dearman R, Kimber I, Griffiths C. Impaired Langerhans cell migration in psoriasis is due to an altered keratinocyte phenotype induced by interleukin-17. Br J Dermatol. 2018;178(6):1364-1372.

87. Liu R, Wang F, Wang Q, Zhao X, Zhang K. Mesenchymal stem cells from skin lesions of psoriasis patients promote proliferation and inhibit apoptosis of HaCaT cells. Genet Mol Res. 2015;14:17758-17767.

88. Seetharaman R, Mahmood A, Kshatriya P, Patel D, Srivastava A. Mesenchymal stem cell conditioned media ameliorate psoriasis vulgaris: a case study. Case Rep Dermatol Med. 2019;2019:987.

89. Zhang Y, Yan J, Li Z, Sun Q. Exosomes derived from human umbilical cord mesenchymal stem cells alleviate psoriasis-like skin inflammation. Int J Mol Sci. 2020.

90. Harman RM, Yang S, He MK, Van de Walle GR. Antimicrobial peptides secreted by equine mesenchymal stromal cells inhibit the growth of bacteria commonly found in skin wounds. Stem Cell Res Ther. 2017;8(1):1-14.

\section{Publish your work in this journal}

Clinical, Cosmetic and Investigational Dermatology is an international, peer-reviewed, open access, online journal that focuses on the latest clinical and experimental research in all aspects of skin disease and cosmetic interventions. This journal is indexed on CAS.
The manuscript management system is completely online and includes a very quick and fair peer-review system, which is all easy to use. Visit http://www.dovepress.com/testimonials.php to read real quotes from published authors. 